\title{
Efeito de oito semanas de treinamento funcional sobre a composição corporal e aptidão física de idosos
} Effect of eight weeks of functional training on
body composition and fitness for elderly

João Paulo Farias¹, Leandro Caique Parreira Póss', Waynne Ferreira Faria, Rui Gonçalves Marques Elias Universidade Estadual do Norte do Paraná (UENP), Jacarezinho, PR, Brasil.

Recebido em: setembro 2015 / Aceito em: novembro 2015 efjoao@hotmail.com

\section{RESUMO}

Objetivo: avaliar o efeito de oito semanas de treinamento funcional sobre a composição corporal e aptidão física de idosos. Método: a amostra foi composta por 10 idosas $(65,33 \pm 4,08$ anos), fisicamente ativas. O programa de exercícios funcionais foi realizado por um período de oito semanas consecutivas. A intensidade do exercício foi determinada por meio da percepção subjetiva de esforço (PSE) de Borg. Os testes utilizados para avaliar a capacidade funcional foram: teste de equilíbrio estático; teste de velocidade de levantar de uma posição sentada; teste de flexão do cotovelo; teste de levantar da cadeira em 30 segundos. A composição corporal foi avaliada através do aparelho de bioimpedância. Resultados: melhoras significativas foram observadas na mobilidade $(p=0,018)$, força de membros superiores $(p=0,000)$ e força de membros inferiores $(p=0,000)$. Com relação a composição corporal, não foram identificadas nenhuma alteração significativa para nenhuma das variáveis analisadas. Conclusão: diante do exposto, conclui-se que oito semanas de treinamento funcional foram eficazes para a melhora da aptidão física de idosos.

Palavras-chave: Envelhecimento; Exercício; Sarcopenia.

\section{ABSTRACT}

Objective: to evaluate the effect of eight weeks of functional training on body composition and physical performance in elderly. Method: the sample consisted of 10 women (65.33 \pm 4.08 years), physically active. The program of functional exercises was conducted for a period of eight consecutive weeks. Exercise intensity was determined by perceived exertion (RPE) of Borg. The tests used to evaluate the functional capacity were: static equilibrium test; Speed up from a sitting position test; elbow bend test; of rising from a chair in 30 seconds test. Body composition was assessed by bioimpedance device. Results: significant improvements were observed in mobility ( $p=0.018$ ), upper limb strength $(p=0.000)$ and lower limb strength $(p=0.000)$. With respect to body composition, no significant changes for any of the variables analyzed were identified. Conclusion: given the above, it is concluded that eight weeks of functional training were effective for improving the physical fitness of elderly.

Keywords: Aging; Exercise; Sarcopenia.

\section{INTRODUÇ̃̃O}

O envelhecimento é descrito como um processo natural e inerente ao ser humano, e que está associado a diversas modificações de cunho biológico, psicológico e funcional. ${ }^{1,2}$ Essas alterações, por sua vez, põem em risco a qualidade de vida do idoso, por limitar a sua capacidade de realizar com vigor, as atividades da vida diária e tornar mais susceptíveis ao desenvolvimento de doenças. ${ }^{3,4}$

Assim, durante esse período a capacidade de pessoas idosas em realizar as atividades da vida diária está muito relacionada com a manutenção dos componentes da aptidão física relacionada à saúde, como produção de força e resistência muscular, equilíbrio, mobilidade, resistência cardiorrespiratória e composição corporal. ${ }^{1}$

Estudos apontam que entre as idades de 30 e 70 anos, ocorre um declínio natural no desempenho dos componentes da aptidão física de indivíduos saudáveis, como a diminuição de $30 \%$ a $50 \%$ da força muscular máxima $^{5}$ e de $20 \%$ a $50 \%$ na flexibilidade dependendo da articulação avaliada. ${ }^{6}$ Além disso, tem sido descrito 
que durante o envelhecimento há uma diminuição na função de diversos sistemas que podem afetar o equilíbrio..$^{2,7}$

Uma das principais causas da diminuição no desempenho motor dessa população está relacionada às alterações na composição corporal, devido ao aumento da adiposidade e, principalmente pela diminuição da massa muscular, também denominada sarcopenia. ${ }^{8,9}$ Neste sentido, estudos tem apontado que a prática regular de atividade física, durante o ciclo de vida de um indivíduo pode atenuar os efeitos da sarcopenia e consequentemente favorecer à manutenção dos componentes da aptidão física relacionada a saúde de idosos. ${ }^{4,10,11}$ Além disso, um posicionamento do American College of Sports Medicine ${ }^{12}$ descreve que, com exceção da restrição calórica, a prática regular de atividade física parece ser o único comportamento de estilo de vida capaz de influenciar positivamente os sistemas fisiológicos e os fatores de risco de doenças crônicas.

Nessa perspectiva, o treinamento funcional tem se destacado como uma alternativa eficaz para a melhora das capacidades físicas de idosos devido ao baixo custo dos aparelhos e a especificidade com as atividades do cotidiano. Entretanto, poucos estudos avaliaram a eficácia deste tipo de treinamento sobre a composição corporal e aptidão funcional de idosos. Assim, o presente estudo teve como objetivo avaliar o efeito da realização de oito semanas de exercícios funcionais, sobre a composição corporal e aptidão física de idosos.

\section{MÉTODO}

\section{Sujeitos}

Participaram deste estudo 10 mulheres idosas $(65,33 \pm 4,08$ anos), fisicamente ativas e que atenderam os seguintes critérios de inclusão: ter mais de 60 anos; não ter nenhum tipo de lesão musculoesquelética e/ou doença que prejudicasse ou impedisse a realização dos testes propostos e apresentar o termo de liberação médica, sendo excluídas aquelas que não atingiram frequência mínima de $75 \%$ das sessões de exercícios.

Todos os participantes, após serem conscientizados sobre a proposta do estudo e procedimentos aos quais seriam submetidos, assinaram o termo de consentimento livre e esclarecido. O estudo foi previamente aprovado pelo comitê de ética local (Parecer CEP número 017/2011, Nº 025/2011).

\section{Avaliação da composição corporal}

O percentual de gordura foi estimado através aparelho de bioimpedância em disposição tetrapolar da marca Maltron ${ }^{\circledR}$, modelo BF-900. As medidas de bioimpedância eram efetuadas uma única vez com o voluntário em decúbito dorsal, em uma maca, sem portar relógio ou qualquer outro objeto metálico. Antes da colocação dos eletrodos na pele do voluntário, era feita limpeza dos pontos de contato com algodão embebido em álcool. As participantes ficavam repousando durante três minutos antes das tomadas de medidas.

\section{Programa de Treinamento}

O programa de exercícios funcionais foi executado por um período de oito semanas consecutivas, compreendendo duas sessões semanais com um in- tervalo mínimo de 48 horas. Todas as sessões foram acompanhadas por um profissional de Educação Física como forma de assegurar a execução correta e segura dos exercícios. Previamente ao início do estudo foram realizadas duas sessões de familiarização dos exercícios a serem executados, com o intuito de aprendizagem das tarefas motoras e adaptação dos aspectos técnicos (intervalo entre os exercícios, velocidade de execução, contagem das repetições). Os exercícios foram realizados em duas rotações em circuito, constituído de nove estações: agachamento livre; serrote com halter; flexão de joelho com a coluna apoiada; crucifixo no colchonete; extensão de quadril; apoiado na parede; elevação lateral; elevação frontal com subida no step; rosca direta e tríceps francês com halter. Cada exercício era realizado de maneira contínua com duração de um minuto e intervalo de 30 s entre cada uma, eram realizados alternados por segmento, na qual cada uma tinha um foco específico em exercícios para membros inferiores e superiores, e exigiam constantemente equilíbrio, controle motor, resistência de força e propriocepção. A intensidade do exercício foi determinada por meio da percepção subjetiva de esforço (PSE) de Borg, ${ }^{13}$ onde foi estabelecida entre moderada à um pouco forte (11-13) como intensidade ótima.

\section{Testes de Capacidade Funcional}

As avaliações das capacidades funcionais foram realizadas no momento inicial e após as oito semanas de treinamento. Na tentativa de minimizar os efeitos intervenientes nas respostas das diferentes variáveis avaliadas (ritmos biológicos naturais), todas as medidas foram realizadas nos mesmos horários pré e pós-treinamento.

Para avaliação da capacidade de equilíbrio foi utilizado o teste de equilíbrio estático sugerido por Matsudo. ${ }^{14}$ Neste teste, o indivíduo inicia em pé, com as mãos na cintura, e após a autorização do avaliador este deve flexionar uma das pernas até a altura do joelho e se manter por pelo menos 30 segundos. São realizadas três tentativas e calculada a média do tempo de execução, sendo permitido ter tentativas iniciais para manutenção da posição.

A mobilidade foi avaliada por meio do teste de velocidade de levantar de uma posição sentada, padronizado por Matsudo. ${ }^{14} \mathrm{O}$ avaliado inicia o teste de uma posição sentada sobre um colchonete, com as pernas juntas e estendidas e com as mãos sobre os joelhos. Ao sinal do avaliador, o indivíduo deve ficar totalmente em pé da maneira que for mais confortável. O cronômetro é acionado simultaneamente a voz de comando e parado no instante em que o avaliado fica totalmente em pé. São executadas três tentativas e sendo considerada o resultado de menor tempo.

A força de membros superiores (FMS) foi avaliada, mediante ao teste, de flexão do cotovelo descrito por Rikli e Jones. ${ }^{15}$ Neste teste o avaliado fica na posição sentada, com as costas eretas e pés apoiados no chão. Em seguida, o peso é colocado na mão dominante, o teste se inicia com o braço totalmente estendido ao lado da cadeira e perpendicular ao solo. Após a autorização do avaliador, o sujeito deve flexionar o braço até completar o ângulo de movimento, tendo um total de 
30s para a realização do máximo de repetições.

O teste de levantar da cadeira em 30s proposto por Rikili e Jones ${ }^{15}$ foi aplicado para avaliar a força de membros inferiores (FMI). O indivíduo inicia o teste na posição sentada em uma cadeira de 43 centímetros, com as costas eretas, pés apoiados no chão e braços frente ao tórax. Após o sinal o avaliado deve se levantar até ficar completamente em pé e retornar à posição inicial, tendo este o tempo de 30s para realizar o máximo de repetições possíveis.

\section{Análise Estatística}

Inicialmente foi utilizado o teste de Shapiro Wilk para verificar a normalidades dos dados. Posteriormente, o teste t de Student foi utilizado para comparações entre os períodos pré e pós para os testes de aptidão e composição corporal. Em todas as análises, a significância adotada foi $p<0,05$, sendo realizadas no pacote estatístico SPSS, versão 22.0.

\section{RESULTADOS}

As características gerais da amostra, bem como a comparação entre os momentos pré e pós-treinamento estão apresentados na tabela 1. Não foi observada nenhuma diferença significativa entre os momentos para as variáveis de composição corporal.

A tabela 2 apresenta os valores e comparações entre os períodos pré e pós-treinamento. Melhoras significativas foram observadas nas variáveis mobilidade $(p=0,018), \operatorname{RMS}(p=0,000)$ e RMI $(p=0,000)$. No entanto, embora não significativa, a variável equilíbrio estático apresentou valores superiores no pós-treinamento em relação ao momento pré.

\section{DISCUSSÃO}

A presente investigação teve como objetivo analisar o efeito do treinamento funcional sobre as variáveis de composição corporal e testes de aptidão funcional de idosos. Os resultados demonstraram que o treinamento funcional foi eficaz para melhora significativa no desempenho dos testes de mobilidade, FMS e FMI. No entanto, não foi observada diferença significativa para nenhuma variável de composição corporal.

Dados na literatura sobre os efeitos do treinamento funcional sobre a composição corporal de idosos são escassos. Comparando os resultados do presente estudo com investigações que prescreveram outras modalidades de treinamento com mulheres idosas, os dados seguem a mesma tendência, no qual apontam que nessa população o exercício físico pode atenuar o declínio da massa muscular e contribuir para um maior controle da gordura corporal. ${ }^{16-19}$ Contudo, devido ao período relativamente curto do treinamento do presente estudo, mudanças significativas na gordura corporal não foram observadas.

Com relação aos testes de aptidão funcional, os resultados estão de acordo com os apresentados por Farias et al., ${ }^{20}$ no qual avaliaram o efeito de oito semanas de treinamento funcional e observaram melhora da mobilidade, FMS e FMI, sem alterações para a variável de equilíbrio. Da mesma forma, Barnett et al. ${ }^{21} \mathrm{e}$ Leal et al. ${ }^{22}$ observaram melhora significativa na força e resistência muscular, equilíbrio e agilidade, após a aplicação de um programa de treinamento funcional. Entretanto, além dos diferentes protocolos de exercícios, nos estudos supracitados as intervenções ocorreram por períodos superiores à presente investigação. Dessa forma, a ausência de significância na variável equilíbrio observada no presente estudo, pode ser explicada em partes pela pouca duração da aplicação do treinamento, haja vista que apesar de não significativo os resultados foram superiores ao momento pré, indicando assim uma tendência a melhora dessa capacidade.

Diversas investigações sugerem que o treinamento com pesos é uma das principais modalidades de exercício físico para melhora e manutenção da capacidade funcional de idosos. ${ }^{12,23-25}$ Contudo, em um estudo recente que comparou o efeito do treinamento com pesos e do treinamento funcional sobre a aptidão física de idosos, Lohne-Seiler, Torstveit e Anderssen ${ }^{26}$ não encontraram diferenças significativas entre o treinamento com pesos e funcional. Além disso, os autores relatam que diferentemente do treinamento com pesos que em grande parte dos exercícios visa trabalhar isoladamente

Tabela 1 - Características gerais da amostra, bem como a comparação entre os momentos pré e pós.

\begin{tabular}{lccc}
\hline & \multicolumn{1}{c}{ Pré } & Pós & P \\
\cline { 2 - 4 } Idade (anos) & $65,33 \pm 5,78$ & & \\
Estatura $(\mathrm{cm})$ & $1,62 \pm 1,35$ & & 0,265 \\
Peso $(\mathrm{kg})$ & $67,38 \pm 10,69$ & $68,83 \pm 11,74$ & 0,613 \\
Massa livre de Gordura (kg) & $40,08 \pm 6,48$ & $41,24 \pm 8,81$ & 0,590 \\
Gordura Corporal (kg) & $28,48 \pm 6,78$ & $27,26 \pm 10,50$ & \\
\hline
\end{tabular}

Tabela 2 - Comparações do desempenho nos testes funcionais pré e pós-treinamento.

\begin{tabular}{lcccc}
\hline & Pré & Pós & \multicolumn{1}{c}{$\%$} & $\mathbf{P}$ \\
\cline { 2 - 5 } Equilíbrio Estático (seg.) & $18,43 \pm 11,61$ & $21,01 \pm 12,11$ & 14,00 & 0,331 \\
Mobilidade (seg.) & $3,91 \pm 1,15$ & $2,93 \pm 1,01$ & $-25,06$ & $0,018^{*}$ \\
RMS (rep.) & $23,00 \pm 3,16$ & $33,90 \pm 4,09$ & 47,39 & $0,000^{*}$ \\
RMI (rep.) & $15,40 \pm 3,02$ & $22,20 \pm 3,58$ & 44,16 & $0,000^{*}$ \\
\hline RMS $=$ Resistêncios
\end{tabular}


cada grupo muscular, o treinamento funcional permite melhorar as capacidades de maneira globalizada com movimentos em diferentes eixos e planos, tornado esse método de treinamento mais dinâmico do que o treinamento com pesos tradicional.

Os resultados do presente estudo indicam que a implantação de programas de treinamento funcional é uma alternativa para melhoria da autonomia funcional de idosos. Contudo, algumas limitações como o pequeno número amostral, período relativamente curto de intervenção, nível de atividade física, falta de controle de hábitos alimentares e ausência do grupo controle dificultam a interpretação dos resultados. Dessa forma, a realização de novos estudos que minimizem essas limitações poderia auxiliar na compreensão dos resultados.

Os resultados do presente estudo evidenciam que oito semanas de intervenção com treinamento funcional foram eficazes para a melhora dos componentes da aptidão física de idosos. Entretanto, não foram identificadas alterações significativas na composição corporal. Recomendamos novos estudos, com maior número amostral.

\section{REFERÊNCIAS}

1. Viana V a R, Esteves AM, Boscolo RA, Grassmann V, Santana MG, Tufik $S$, et al. The effects of a session of resistance training on sleep patterns in the elderly. Eur J Appl Physiol [Internet] 2012; 112(7): 2403-8. DOI: http://dx.doi. org/10.1007/s00421-011-2219-2.

2. Holviala J, Kraemer WJ, Sillanpää E, Karppinen $H$, Avela J, Kauhanen a, et al. Effects of strength, endurance and combined training on muscle strength, walking speed and dynamic balance in aging men. Eur J Appl Physiol [Internet] 2012; 112(4): 1335-47. DOI: http://dx.doi.org/10.1007/s00421-011-2089-7.

3. Mosallanezhad Z, Salavati M, Sotoudeh GR, Nilsson Wikmar $\mathrm{L}$, Frändin $\mathrm{K}$. Walking habits and health-related factors in 75-year-old Iranian women and men. Arch Gerontol Geriatr [Internet] 2014; 58(3): 320-6. DOI: http://dx.doi. org/10.1016/j.archger.2013.11.008.

4. Matsudo SM, Matsudo VR, Barros Neto TL. Atividade física e envelhecimento : aspectos epidemiológicos. Rev Bras Med do Esporte 2001; 7(1): 2-13. DOI: http://dx.doi.org/10.1590/ S1517-86922001000100002.

5. Jozsi AC, Campbell WW, Joseph L, Davey SL, Evans WJ. Changes in power with resistance training in older and younger men and women. Journals Gerontol [Internet] 1999; 54(11): M591-6.

6. Holland GJ, Tanaka K, Shigematsu R, Nakagaichi M. Flexibility and physical functions of older adults: A review. J Aging Phys Act 2002; 10: 169-206.

7. Ricci NA, Gazzola JM, Coimbra IB. Sistemas sensoriais no equilíbrio corporal de idosos Sensory. Arq Bras Ciências da Saúde 2009; 34(2): 94-100.

8. Araujo Silva TA, Frisoli Junior A, Pinheiro MM, Szejnfeld VL. Sarcopenia Associada ao Envelhecimento: Aspectos Etiológicos e Opções Terapêuticas. Rev Bras Reumatol 2006; 46(6): 391-7. DOI: http://dx.doi.org/10.1590/S048250042006000600006.

9. Pierine DT, Nicola M, Oliveira EP. Sarcopenia: alterações metabólicas e consequências no envelhecimento. Rev Bras Ciência e Mov 2009; 17(3): 96-103.

10. Marques E A, Baptista F, Santos D A, Silva AM, Mota J, Sardinha LB. Risk for losing physical independence in older adults: The role of sedentary time, light, and moderate to vigorous physical activity. Maturitas [Internet]. Elsevier Ireland Ltd 2014; 8-12. DOI: http://dx.doi.org/10.1016/j. maturitas.2014.06.012.

11. Garber CE, Blissmer B, Deschenes MR, Franklin B a, Lamonte MJ, Lee I-M, et al. Quantity and quality of exercise for developing and maintaining cardiorespiratory, musculoskeletal, and neuromotor fitness in apparently healthy adults: guidance for prescribing exercise. Med Sci Sports Exerc [Internet] 2011; 43(7): 1334-59. DOI: http:// dx.doi.org/10.1249/MSS.Ob013e318213fefb.

12. Chodzko-Zajko WJ, Proctor DN, Fiatarone Singh M a, Minson CT, Nigg CR, Salem GJ, et al. American College of Sports Medicine position stand. Exercise and physical activity for older adults. Med Sci Sports Exerc [Internet] 2009; 41(7): 1510-30. DOI: http://dx.doi.org/10.1249/MSS.0b013e3181a0c95c.

13. Borg G. An Introduction to Borg's RPE-Scale. Movement Publications, Ithaca, NY. New York; 1985.

14. Matsudo SM. Avaliação do idoso: física e funcional. $2^{\mathrm{a}} \mathrm{Ed}$. Midiograf, editor. Londrina, Pr; 2004.

15. Rikli RE, Jones JC. Development and validation of a functional fitness test for community-residing older adults. J Aging Phys Act 1999; 7(2): 129-61. DOI: http://dx.doi. org/10.1093/geront/gns071.

16. Raso V. Exercícios com pesos para pessoas idosas : a experiência do Celafiscs. Rev Bras Ciência e Mov 2000; 8(2): 41-9.

17. Silva CM, Gurjão ALD, Ferreira L, Gobbi LTB, Gobbi S. Efeito do treinamento com pesos, prescrito por zona de repetições máximas, na força muscular e composição corporal em idosas. Rev Bras Cineantropometria Desempenho Hum 2006; 8(4): 39-45.

18. Ferreira MT. O papel da atividade física na composição corporal de idosos. Rev Bras Ciências da Saúde 2003; 1(1): 43-52. DOI: http://dx.doi.org/10.13037/rbcs.vol1n1.495.

19. Melo GF De, Giavoni A. Comparação dos efeitos da ginástica aeróbica e da hidroginástica na composição corporal de mulheres idosas. Rev Bras Ciência e Mov 2004; 12(2): 13-8.

20. Farias JP, Scatambuli T, Silva FV, Faria WF, Santos CF, Marques Elias RG. Efeito de oito semanas de treinamento funcional sobre a aptidão funcional de idosos. Acta Bras do Mov Hum 2014; 4(1): 13-27.

21. Barnett A, Smith B, Lord SR, Williams M, Baumand A. Community-based group exercise improves balance and reduces falls in at-risk older people: a randomised controlled trial. Age Ageing [Internet] 2003; 32(4): 407-14.

22. Leal SM, Borges EG, Fonseca MA, Alves Junior E, Cader S, Dantas EHM. Efeitos do treinamento funcional na autonomia funcional, equilíbrio e qualidade de vida de idosas. Rev Bras Ciência e Mov 2010; 17(3): 61-9.

23. Dias RMR, Gurjão ALD, Marucci M de FN. Benefícios do treinamento com pesos para aptidão física de idosos. Acta Fisiátrica 2006; 13(2): 90-5.

24. Best JR, Nagamatsu LS, Liu-Ambrose T. Improvements to executive function during exercise training predict maintenance of physical activity over the following year. Front Hum Neurosci [Internet] 2014; 8(May): 353. DOI: http://dx.doi.org/10.3389/fnhum.2014.00353.

25. Barros KD De, Oliveira AAB De, Oliveira Filho A De. A influência do treinamento com pesos em mulheres acima de 50 anos. Acta Sci Heal Sci [Internet] 2011; 33(1): 43-50. DOI: http://dx.doi.org/10.4025/actascihealthsci.v33i1.7619.

26. Lohne-Seiler H, Torstveit MK, Anderssen SA. Traditional versus functional strength training: Effects on Muscle Strength and Power in the Elderly. Aging Phys Act 2013; 21: 51-70. 